\title{
European Commission Proposes Radical Overhaul of Animal Transport Rules
}

In an effort to minimize the stress that animals experience during transportation, the European Commission (EC) on 16 July 2003, adopted a proposed Regulation that will radically overhaul the animal transport rules in Europe ${ }^{1}$. "To improve enforcement, the Regulation identifies the chain of all those involved in animal transport and who is responsible for what, as well as introducing efficient enforcement tools, such as checks via the tachograph. It also introduces much stricter rules for journeys of more than 9 hours, including domestic transport which mirror other EU legislation governing the time that drivers

\section{Regulation Update}

OLAW, APHIS Revise Guidance Regarding IACUC Approval of Changes in Personnel Involved in Animal Activities

On 6 June, the Office of Laboratory Animal Welfare (OLAW), in consultation with the USDA Animal and Plant Health Inspection Service (APHIS), Animal Care, amended guidance provided in 1995 to Public Health Service (PHS) awardee institutions and IACUCs concerning approval of changes in personnel involved in animal activities ${ }^{2}$. Such changes were considered major by OLAW in the past and therefore required full-committee or designated-member review under the provisions of the PHS Policy and the USDA animal welfare regulations. The revised guidance allows such changes to be handled as follows:

"IACUCs may, by institutional policy, classify certain proposed additions and changes in personnel, other than the Principal Investigator, as 'minor' provided that an appropriate administrative review mechanism is in place to ensure that all such personnel are appropriately identified, adequately trained and qualified, enrolled in applicable occupational health and safety programs, and meet other criteria as required by the IACUC. The IACUC remains responsible for confirming that all IACUC review criteria regarding personnel training and qualifications are maintained and documented (PHS Policy IV,C, 1 and 9CFR 1, chapter 1, Part 2, 2.31). Institutions will be held accountable for ensuring the performancebased outcome that all individuals involved in animal-related activities are competent to do so. This guidance is intended to provide substantial reduction of burden on IACUCs and research teams without diminishing the crucial oversight of personnel training and qualifications²."

can spend on the road. The proposed Regulation recognizes that most of the stress on the animals occurs around loading and unloading and therefore introduces rules to deal with situations before and after transport, for example at slaughterhouses or at harbours. It encourages Member States to develop guides of good practice."

David Byrne, Commissioner for Health and Consumer Protection, said in a press release, "I am optimistic that this radical overhaul of the animal transport rules in Europe can come into force soon. I believe it provides the best tools to introduce real improvements in animal welfare over the short to medium term. Importantly, the Regulation will also define who is responsible for what and when, which will help enormously in ensuring that the rules are properly enforced ${ }^{1} . "$

The new Regulation reduces the maximum travel times for all species and forbids the transportation of particularly young or pregnant animals. Likewise, it calls for improved traveling conditions, including species-appropriate temperatures, permanent access to drinking water, and increased minimums for space according to species and length of journey ${ }^{1}$.

The proposed Regulation increases the list of people responsible for animal transportation conditions and increases personal responsibility in case of infringement.

The Regulation will have to be approved by the Council of Ministers after consultation of the European Parliament. It is expected to be in effect by the end of 2005 .

\section{References}

1. European Commission. Commission proposes radical overhaul of animal transport rule. (16 July 2003). http://europa.eu.int/ $\mathrm{rapid} / \mathrm{start} / \mathrm{cgi} / \mathrm{guesten} . \mathrm{ksh}$ ?p_action. gettxt=gt\&doc=IP/03/1023 $\mid$ 0|RAPID\&Ig=EN \&display.

2. NIH. Office of Extramural Research revised guidance regarding IACUC approval of changes in personnel involved in animal activities. Notice: NOT-OD-03-046. (6 June 2003). http://grants1.nih.gov/grants/guide/ notice-files/NOT-OD-03-046.html. 